\title{
A New Color Information Entropy Retrieval Method for Pathological Cell Image
}

\author{
Xiangang Jiang ${ }^{1}$, Qing Liang ${ }^{2}$, and Tao Shen ${ }^{2}$ \\ ${ }^{1}$ Information Engineering School of East China, Jiaotong University, \\ Nanchang, Jiangxi, China (330013) \\ jxg_2@tom.com \\ ${ }^{2}$ Basic Science School of East China \\ Jiaotong University, Nanchang, Jiangxi, China (330013) \\ LiangQing1987@163.com
}

\begin{abstract}
For the distribution characteristics in a slice of pathological cell image, the system transforms them into the characteristic vector by quantization and clustering in HSV color model, it promotes the concerned isolated pixel color description into the color feature of its neighborhood region color histogram. By choosing appropriate neighborhood window size, it uses color information entropy method to gain efficient primary classification of gastric slice image and the similar retrieval results from the sample library images. Experiments show that this method has a better image classification and retrieval result.
\end{abstract}

Keywords: Mutual information, Histogram, Color moments, Information entropy, CBIR.

\section{Introduction}

With the unceasing development of the modern imaging technology and information processing technology, the image numbers in medical scopes are more and more. By the submitting image, how to use the existing technical methods to retrieve and classify the comparative images from the qualitative sample library is crucial, which is used for clinical diagnosis and contrast, it is becoming one of the research task for efficient medical diagnosis. In allusion to the pathological cell images, this paper makes use of neighborhood color moment histogram to extract color information and utilizes the theory of information entropy to realize shortcut image retrieval.

\section{Shannon Mutual Information}

Representation of mutual information derives from information theory, which is the measure of correlations of two variables. If use $H$ to represent entropy, $I$ represent an image, $a$ represent the color values of an image, suppose the probability density 
function of $I$ is $p_{i}=p(a=i), i=1,2, \ldots, m$, then the Shannon Entropy of $I$ is $H(I)$ :

$$
H(I)=\sum_{i \in I} p_{i}(i) \times \log \frac{1}{p_{i}(i)}
$$

Where as $p_{i}(i)=0$, supplementary definition:

$$
p_{i}(i) \times \log \frac{1}{p_{i}(i)}=0 \text {, as: } \lim _{p \rightarrow 0^{+}} p \log \frac{1}{p}=0 \text {. }
$$

To two images $I_{1}$ and $I_{2}$, their color values are described by 256 kinds of vectors, their joint entropy is defined as:

$$
H\left(I_{1}, I_{2}\right)=\sum_{i=0}^{255} \sum_{j=0}^{255} P_{i j}(i, j) \log \frac{1}{P_{i j}(i, j)}
$$

Where $P_{i j}(i, j)$ is the representation of color joint probability density distribution.

Their Shannon mutual information $I\left(I_{1}, I_{2}\right)$ is defined as:

$$
\begin{aligned}
I\left(I_{1}, I_{2}\right) & =\sum_{i=0}^{255} \sum_{j=0}^{255} P_{i j}(i, j) \log \frac{P_{i j}(i, j)}{p_{i}(i) p_{j}(j)} \\
& =H\left(I_{1}\right)+H\left(I_{2}\right)-H\left(I_{1}, I_{2}\right)
\end{aligned}
$$

Where $p_{i}(i), p_{j}(j)$ is the color probability density distribution of $I_{1}$ and $I_{2}, P_{i j}(i, j)$ is color joint probability density distribution, $H\left(I_{1}, I_{2}\right)$ is Shannon joint entropy. In the image registration and retrieval, Shannon mutual information is a representation of information amount of interactivity implicated in two images. When $I\left(I_{1}, I_{2}\right)=0$, it shows that $I_{1}$ and $I_{2}$ are independent mutually, if the value of $I\left(I_{1}, I_{2}\right)$ is bigger, which means the similarity is higher in the color distribution of two images.

\section{Color Space Conversions}

Among many color models, RGB models can be better matched with the fact that people can fiercely percept the tricolor such as $\mathrm{R}, \mathrm{G}$, and $\mathrm{B}$, furthermore, it is easy to express in software developing language. However, RGB color model is not accorded to the human beings' visual perception dealing with distinguishing color similarity. Therefore, many researchers put forward some other color models accorded to visual perception, such as HIS, HSV, Luv, Lab and so on. HSV is more commonly used, its three-component separately represent Hue, Saturation and Value. This paper uses the HSV color model to define color vector. Firstly the transfer formula between RGB and HSV is given as follows. Suppose a pixel values are $r$, $g$, and b in RGB color model, in 
which $r, g, b \in[0 \ldots 255]$, then the transformed values (h,s and v) in HSV color model are calculated as follows : Suppose $v^{\prime}=\max (r, g, b)$ and define $r^{\prime}, g^{\prime}, b^{\prime}$ as follows:

$$
r^{\prime}=\frac{v^{\prime}-r}{v^{\prime}-\min (r, g, b)}, \quad g^{\prime}=\frac{v^{\prime}-g}{v^{\prime}-\min (r, g, b)}, \quad b^{\prime}=\frac{v^{\prime}-b}{v^{\prime}-\min (r, g, b)}
$$

Then $\quad v=v^{\prime} / 255$,

$$
\begin{gathered}
s=\left(v^{\prime}-\min (r, g, b)\right) / v^{\prime} \\
h^{\prime}=\left\{\begin{array}{l}
5+b^{\prime}, r=\max (r, g, b) \text { and } g=\min (r, g, b) \\
1-g^{\prime}, r=\max (r, g, b) \text { and } b=\min (r, g, b) \\
1+r^{\prime}, g=\max (r, g, b) \text { and } b=\min (r, g, b) \\
3-b^{\prime}, g=\max (r, g, b) \text { and } g=\min (r, g, b) \\
3+g^{\prime}, b=\max (r, g, b) \text { and } r=\min (r, g, b) \\
5-r^{\prime}, \text { else }
\end{array}\right. \\
h=60 \times h^{\prime}
\end{gathered}
$$

Where $r^{\prime}, g^{\prime}, b^{\prime} \in[0,1], h \in[0,360], s, v \in[0,1]$.

In allusion to Fig.1, make use of the transfer formula above firstly, in the HSV color model $\mathrm{h}$ component is mainly concentrated in range $[0,100]$ and $[300,360]$, but the distribution of $\mathrm{s}$ and $\mathrm{v}$ component are relative uniformity. According to the characteristics, it will use the method in reference [1] for quantization and clustering of colors. Suppose the color vectors of pixel $(i, j)$ are $p_{i j} \in[0,255]$, which will be used for image retrieval features.

\section{Neighborhood Color Moment Mutual Information}

\subsection{Description of Color Moment}

A. single pixel color moment

Another method of expressing and analyzing color distribution is the calculation of color moment. The mathematical foundation of this method being reasonable is that any color distribution can be represented by its moments. In addition, as the color distribution information is mainly concentrated in lower-order-moments, so it is enough to express the color distribution of an image only by its first moment, second moment and third moment of colors. Comparing to color histogram, this method is good at the aspect that no needs to quantize the color characteristics. The lower-order-moments of color are expressed in mathematics as follows: 


$$
\left\{\begin{array}{l}
\mu=\frac{1}{N_{A}} \sum_{j=1}^{N} p_{j} \\
\sigma=\left(\frac{1}{N_{A}} \sum_{j=1}^{N}\left(p_{j}-\mu\right)^{2}\right)^{\frac{1}{2}} \\
s=\left(\frac{1}{N_{A}} \sum_{j=1}^{N}\left(p_{j}-\mu\right)^{3}\right)^{\frac{1}{3}}
\end{array}\right.
$$

Where $p_{j}$ is the definition of color value of pixel $j, N_{A}$ represents the whole number of pixels in an image.

B. Neighborhood color moment

As the ability to distinguish based on lower-order-moments of single pixel color is suboptimal, it doesn't make comprehensive consideration to its local color distribution of pixels. So in this paper, it considers the neighborhood of pixels as an analysis unit to calculate the corresponding color moments and it concerns the local color spatial distribution information reflected by pre-three lower-order-moments in a pixel's around window. Suppose $p_{i j}$ is the color value of concerned pixel $(i, j)$, which is quantified and clustered in HSV color model, then its first, second and third order central moment are as follows:

$$
\left\{\begin{array}{l}
\mu_{i j}^{M}=\frac{1}{M \times N} \sum_{m=i-\frac{N-1}{2}}^{i+\frac{N-1}{2}} \sum_{n=j-\frac{M-1}{2}}^{j+\frac{M-1}{2}} p_{m n} \\
\sigma_{i j}^{M}=\left[\frac{1}{M \times N} \sum_{m=i-\frac{N-1}{2}}^{i+\frac{N-1}{2}} \sum_{n=j-\frac{M-1}{2}}^{j+\frac{M-1}{2}}\left(p_{m n}-u_{i j}^{M}\right)^{2}\right]^{\frac{1}{2}} \\
s_{i j}^{M}=\left[\frac{1}{M \times N} \sum_{m=i-\frac{N-1}{2}}^{i+\frac{N-1}{2}} \sum_{n=j-\frac{M-1}{2}}^{j+\frac{M-1}{2}}\left(p_{m n}-u_{i j}^{M}\right)^{3}\right]^{\frac{1}{3}}
\end{array}\right.
$$

The moving window is respectively selected as $3 \times 3,5 \times 5,7 \times 7$, the range of numerical values in extraction of three lower-order-moments of all pixels $(i, j)$ is limited in $[0,255]$. In allusion to each pixel in an image, it makes use of quantization and clustering color histogram of image referred above to obtain nine neighborhood color moments, (for a window $3 \times 3$ ), which are described in the region $[0,255]$ just by rounding all float numbers. It makes the global statistics and normalization to the entire image and gets normalization histograms of nine neighborhood lower-order central moments.Fig. 2 shows as: the normalization histograms of the first, second and third order central moment in $3 \times 3$ neighborhood window, where the red curve represents $H\left(\mu^{3}\right)$, the green one represents $H\left(\sigma^{3}\right)$, the blue one represents $H\left(s^{3}\right)$ and the black one represents the color histogram by quantified and clustered color vectors. 


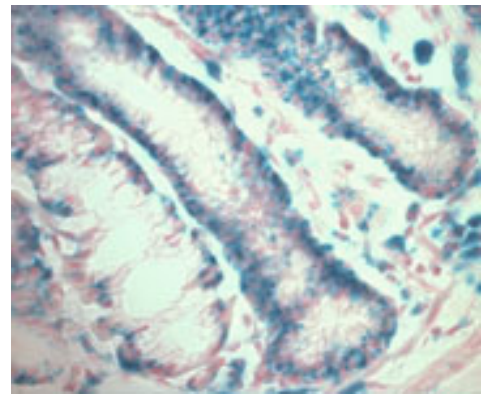

Fig. 1. Pathological cell image

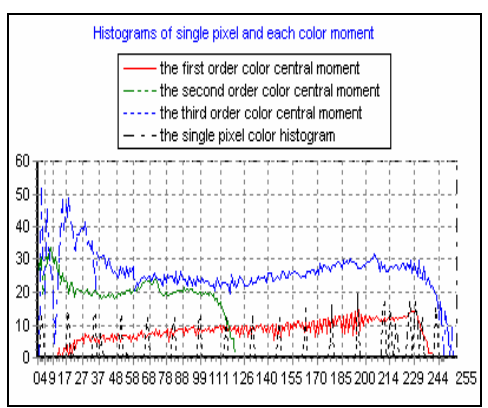

Fig. 2. The compared histograms of the first, second, third order moment and the entire image's quantified and clustered color vectors in the $3 \times 3$ neighborhood window

To 256 colors in the picture, it can make a statistics separately for the frequency of the first, second and third order central moment in an image, suppose the probability distribution of the k order central moment is $q_{i k}$, then:

$$
q_{i k}=N_{i k} / N_{A}
$$

Where $N_{i k}$ represents the occurrence times of the k order color central moment, $N_{A}$ is the representation of all pixel numbers in an image. Define the color joint probability density distribution of the $\mathrm{k}$ order central moment of two images by the same methods, which is supposed as $q_{i j k}$, where $i, j=0,1, \ldots 255, k=1,2,3$.

\subsection{The Calculation of Mutual Information of Each Order Multi-neighborhood}

\section{Color Moments}

In order to define the mutual information of multi-neighborhood color moments, instead $q_{i k}, q_{i j k}$ of $p_{i}(i), P_{i j}(i, j)$ to obtain the definition of entropy and mutual information of multi-neighborhood color moments.

Then the definition of entropy of each order multi-neighborhood color moments is:

$$
H(I)=\sum_{i \in I} q_{i k}(i) \times \log \frac{1}{q_{i k}(i)} \quad(k=1,2,3)
$$

The mutual information of each order multi-neighborhood color moments between two images $I_{1}, I_{2}$ is defined as:

$$
\begin{aligned}
I\left(I_{1}, I_{2}\right) & =\sum_{i=0}^{255} \sum_{j=0}^{255} q_{i j k}(i, j) \log \frac{q_{i j k}(i, j)}{q_{i k}(i) q_{j k}(j)} \quad(k=1,2,3) \\
& =H\left(I_{1}\right)+H\left(I_{2}\right)-H\left(I_{1}, I_{2}\right)
\end{aligned}
$$


In all, the classification and retrieval for the pathological cell images can be achieved not only by the mutual information of each order multi-neighborhood color moments, but also by the mutual information of synthesis vector normalized by each order neighborhood color moment.

\subsection{Similarity Definition}

According to the theory of mutual information, the similarity of two images is defined as follows:

$$
S\left(I_{1}, I_{2}\right)=\frac{I\left(I_{1}, I_{2}\right)}{H\left(I_{1}\right)+H\left(I_{2}\right)}
$$

Proof, $0 \leq S\left(I_{1}, I_{2}\right) \leq 1$. First, because of nonnegativity of mutual information and the definition of information entropy referred, it shows that $I\left(I_{1}, I_{2}\right) \geq 0, H\left(I_{1}\right) \geq 0$, $H\left(I_{2}\right) \geq 0$,each term of numerator and denominator in formula(10) is nonnegative, therefore, $S\left(I_{1}, I_{2}\right) \geq 0$. Next prove $S\left(I_{1}, I_{2}\right) \leq 1$, Because of the definition of color entropy, it shows $H\left(I_{1}, I_{2}\right) \geq 0$ and it can be easy to know $I\left(I_{1}, I_{2}\right) \leq H\left(I_{1}\right)+H\left(I_{1}\right)$ according to formula(9), and then $S\left(I_{1}, I_{2}\right) \leq 1$.So, on the basis of the analysis above, The function $S\left(I_{1}, I_{2}\right)$ satisfies the definition of similarity, it can be used to describe the color similarity measure of two images.

\section{The Experimental Analysis and Conclusions}

In allusion to three hundred images downloaded from www.phoenixpeptide.com $\& w w w . m i c r o s c o p y u . c o m$, sixty cancer images are included and the other ones are all normal. It clearly shows that the main distinctions between cancer images and normal ones are the differences of their color distribution by comparing and analyzing their information entropy of color moment. In the experiment, it employs a new color information entropy retrieval method which promotes the concerned isolated pixel color description into the color feature of its neighborhood region color histogram and makes use of the theory of information entropy to gain the retrieval and primary classification to a further compared classification between the key image and the qualitative sample library. There are three test patterns designed in this experiment the first one do an experiment of retrieval based on color mutual information of single pixel; the second one do an experiment of retrieval by making use of the isolated mutual information of the pre-three order color moment in window size of $3 \times 3$, $5 \times 5,7 \times 7$ separately; the third one: do an experiment of retrieval based on the mutual information of the normalized characteristics vector of the three order color moments. This system uses the method called "based on the key image" that it gives a submitting image and finds out the similar images from the sample image library, while 
a retrieval process finished, the system will return the most similar eight images from the qualitative sample image library to achieve the primary qualitative analysis between the submitting image and the qualitative images from the sample image library. This experiment adopts image shown in Fig.1 as the key image.

This system implements the retrieval and classification of stomach cell images in a development environment of Windows XP by Delphi 7.0, precision is used for the estimation of the experimental efficiency and it is defined as:

$$
\operatorname{Pr} \text { ecision }=\frac{a}{a+b}
$$

Where ' $a$ ' represents the similar image numbers with the key image retrieved from the sample image library; ' $b$ ' represents the image numbers of dissimilarity.

In allusion to the image in Fig. 1, it uses the method of the mutual information of the single pixel to achieve retrieval and obtain the most similar eight images are showed in Fig.3, Fig.4 shows the retrieval results by employing the method based on the mutual information of the first order color moment in the window size of $5 \times 5$. Each color mutual information retrieval efficiency are showed as the table 1.

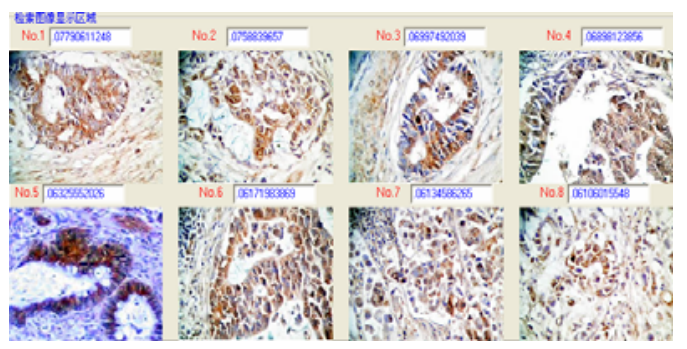

Fig. 3. The retrieval result by the method based on mutual information of a single pixel

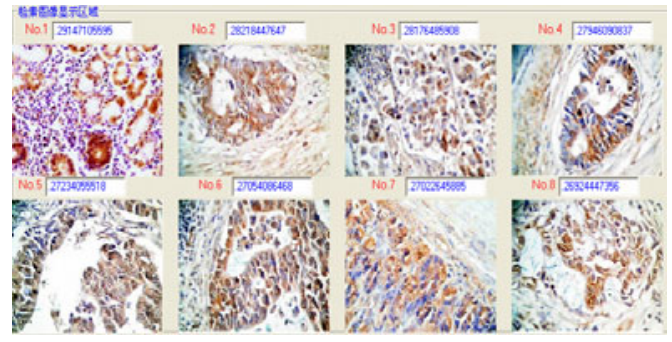

Fig. 4. The retrieval result by the method based on mutual information of the first order color moment with a window size of $5 \times 5$ 
Table 1. The comparison of each method of color mutual information

\begin{tabular}{|c|c|c|c|c|c|}
\hline \multicolumn{2}{|c|}{ Retrieval method } & \multirow{2}{*}{$\begin{array}{l}\text { Precision } \\
\qquad(\%)\end{array}$} & \multirow{3}{*}{$\begin{array}{l}\text { Precision of } \\
\text { using three } \\
\text { order color } \\
\text { moment } \\
\text { synthesis (\%) }\end{array}$} & \multirow{2}{*}{$\begin{array}{l}\text { Consuming } \\
\text { time }(\mathrm{ms})\end{array}$} & \multirow{3}{*}{$\begin{array}{l}\text { Consuming time of } \\
\text { using three order } \\
\text { color moment } \\
\text { synthesis (ms) }\end{array}$} \\
\hline \multirow[t]{2}{*}{$\begin{array}{l}\text { Size of } \\
\text { window }\end{array}$} & $\begin{array}{l}\text { Types of color } \\
\text { mutual } \\
\text { information }\end{array}$ & & & & \\
\hline & $\begin{array}{l}\text { Single pixel } \\
\text { color }\end{array}$ & 80.65 & & 6141 & \\
\hline \multirow{3}{*}{$3 * 3$} & $\begin{array}{l}\text { The first order } \\
\text { color moment }\end{array}$ & 88.25 & \multirow{3}{*}{91.23} & 8828 & \multirow{3}{*}{18012} \\
\hline & $\begin{array}{l}\text { The second order } \\
\text { color moment }\end{array}$ & 90.19 & & 8875 & \\
\hline & $\begin{array}{l}\text { The third order } \\
\text { color moment }\end{array}$ & 90.19 & & 9234 & \\
\hline \multirow{3}{*}{$5 * 5$} & $\begin{array}{l}\text { The first order } \\
\text { color moment }\end{array}$ & 87.88 & \multirow{3}{*}{92.56} & 8625 & \multirow{3}{*}{26412} \\
\hline & $\begin{array}{l}\text { The second } \\
\text { order color } \\
\text { moment }\end{array}$ & 91.30 & & 12375 & \\
\hline & $\begin{array}{l}\text { The third order } \\
\text { color moment }\end{array}$ & 92.65 & & 13025 & \\
\hline \multirow{3}{*}{$7 * 7$} & $\begin{array}{l}\text { The first order } \\
\text { color moment }\end{array}$ & 89.31 & \multirow{3}{*}{93.17} & 8968 & \multirow{3}{*}{36247} \\
\hline & $\begin{array}{l}\text { The second } \\
\text { order color } \\
\text { moment }\end{array}$ & 92.45 & & 16875 & \\
\hline & $\begin{array}{l}\text { The third order } \\
\text { color moment }\end{array}$ & 92.86 & & 17400 & \\
\hline
\end{tabular}

From the above table, we know that the retrieval efficiencies of the methods of mutual information of each order color moment regarding neighborhood region are higher than the one based on the single pixel, meanwhile, the retrieval efficiencies relative to the color moment order trend to be positive growth, the way based on synthesis mutual information of each order neighborhood color moment has the best precision. At the point of consuming-time, the method of each color moment is longer relatively to the one based on the single pixel and it is in direct proportion to the order. In allusion to the retrieval method of mutual information of color moment in different 
neighborhood sizes, while the geometric size of each tissue in an image is small, it will be better to choose the smaller window neighborhood, which has a high retrieval efficiency and low consuming-time; otherwise select a bigger neighborhood window to the bigger tissue in an image, it would avoid the characteristic fuzzy of each tissue in an image by adopting a flexible window size.

\section{References}

[1] Chen, Q., Tai, X., Ba, T.: Gastroscope image retrieval based on histogram of neighborhood color moments. J. Computer Engineering and Applications 44(11), 205-208 (2008)

[2] Wang, Y.: Expression on the Basis of the Timber Surface Color Characteristic of the Histogram and Color Moment Method. J. Forestry Science \& Technology 31(5), 56-58 (2006)

[3] Dong, W., Zhou, M., Geng, G.: Image Retrieval Technique Based on combined features. J. Computer Applications and Software 22(10), 34-36 (2005)

[4] Fan, Z., Liu, E., Xu, B.: Application of Mutual Information in Image Retrieval. J. Journal of University of Electronic Science and Technology of China 36(6), 1311-1314 (2007)

[5] Huang, X., Chen, G.: Color image retrieval based on color and texture. J. Popular Science \& Technology 126(2), 13-15 (2010) 